\title{
An ODA/Dexter hyperdocument system with automated link definition
}

\author{
M. Worring, R. Buitenhuis, A.W.M. Smeulders \\ Department of Computer Systems, University of Amsterdam \\ Kruislaan 403, 1098 SJ Amsterdam, The Netherlands \\ $\mathrm{Tel}+31205257521, \mathrm{Fax}+31205257490$ \\ e-mailworring@fwi.uva.nl
}

\begin{abstract}
In this contribution we consider the construction and storage of hyperdocuments; converting scanned paper documents into hypertext. Hyperlink generation is automated by analyzing the image of the document. A number of document analysis tools are described. For the hyperlink detection the main contribution of this paper is the automated construction of links between the text and picture labels. The storage and retrieval of documents is based on the recently developed Dexter Hypertext Reference Model [4] in conjunction with the well known Open Document Architecture [1]. The symbiosis of these two standards leads to a solid base for data structures and data manipulation. Some practical results of hyperlink acquisition are presented.
\end{abstract}

\section{Keywords}

Hypertext, dexter, document analysis, OCR, ODA, picture labels, icon detection

\section{INTRODUCTION}

Due to increased storage capacity and network performance the storage and distribution of large collections of scanned paper documents has become feasible. Document information systems are usually constructed by "image enabling" a standard DBMS. Retrieval of the scanned documents is then on the basis of predefined identification labels only and the functionality added to the database system is limited to the display of the document.

A document can contain text and/or pictures and graphics and it would be far more appropriate to retrieve the different document parts on the basis of their content; for example full text retrieval of documents on the basis of user specified keywords or the selection of all graphical pictures containing a specific visual icon. At this point it should be noted that we only have an image of the document. In order to allow for the above type of queries we need to analyze the image to find the corresponding text and detect visual icons in the pictures. The first step in this analysis is to classify automatically different parts of the image as text blocks, photos, tables etc. Blocks classified as text can then be 
passed through an Optical Character Recognition (OCR) program to retrieve the ASCII text conveyed by the image of the text block. Apart from revealing the content of the document, the text analysis also leads to significant data reduction as ASCII codes are far more compact than the corresponding image data.

The appropriate data structure for storing the structure of the document is the Open Document Architecture (ODA) [1]. This data structure considers both the geometric and the logical structure of the document.

Documents can now be retrieved on the basis of the content of the different blocks or on the geometric and logical structure of the document. However, browsing through a large collection of blocks does not only require to consider the content of individual blocks but also their relations. Retrieval then becomes "jumping" from one document part to related document parts. This requires a hypertext system, providing item-to-item (hyper)links, where each item can be a piece of text, a picture, or a picture detail.

Most hypertext systems contain text-text or text-picture links only. However, links between text and picture details are very important as well. For example in patent applications or manuals, labels in the picture identify components which are described in the accompanying text. Such links are particularly convenient in complicated documents with a large number of numeric labels where finding a specific label by visual inspection is a laborious task. Furthermore, in pictures with a legend (for example tourist maps) links between a specific legend item and the positions of this item in the map are of great importance for quick browsing.

Construction of hyperdocuments from scanned documents by interactive definition of relational links is a tedious task. However, many links can be generated automatically. For example in [5] and [8], automatic text-text link definition is performed by using OCR. In [6] a system is presented where links between words in the text and the corresponding pictures as a whole are detected. The linkage types used in the references are limited. Especially the links with picture details are missing. In this contribution we consider a system for hypertext construction from documents which aids the user in the generation of all of the above mentioned linkage types.

Due to the inherent complexity of a hyperdocument system with such a complicated linkage structure it is important to carefully consider the data structures for storing the hyperdocument. We therefore base our system on the Dexter Hypertext Reference Model [4] which is a recently defined model for both describing current hypertext systems as well as a useful tool in the design of hypertext systems. In fact the model is a superset of the functionality of commonly available hypertext systems.

The reference model deals with the structure of links in a hypertext system only. It explicitly avoids defining the structure within the documents which has to be described by other models. As noted before, ODA provides a convenient way of structuring the contents of the documents. As will be shown later the ODA structure is easily incorporated into a Dexter Hypertext System. It also provides a proper basis for the document analysis.

This paper is organized as follows. First an introduction to document structure as defined in ODA is given and the set of modules for analyzing the document and its contents are introduced (section 2). Then in section 3, different types of links are analyzed and methods for finding them, based on the document analysis modules, are presented. In section 4 we consider the incorporation of the ODA model into the Dexter Model. Finally, we show results of automated link generation in section 5 . 


\section{DOCUMENT STRUCTURE AND ANALYSIS}

In this section we introduce the Open Document Architecture, which will be used later for the storage of documents and in the document analysis phase.

\subsection{The Open Document Architecture}

The Open Document Architecture [1] is an international standard for the representation of structured documents. It is designed to provide a standard for storing, editing and interchange of documents. In ODA two different types of structures are distinguished. First, the geometric structure which gives the structure of the document the way it is presented and the logical structure giving meaning to the different parts of the document. The smallest components considered are the basic geometric or logical objects. Such an object can, for example, be a piece of text or a picture. A geometric and logical object are connected via their content. The geometric object describes at which position the object is located, in case of text the fontsize used etc. The logical object describes the meaning of the object, for example the piece of text being a header or a paragraph.

Basic components can be grouped in frames. A section frame, for example, contains several paragraphs and headers for subsections. The ODA structure further defines pages and combines them into page-sets.

The geometric and logical structure of a document can both be described as trees where the basic objects are the leaves of the tree and the intermediate nodes are either frames or pages. For a given document the trees define the specific geometric structure and the specific logical structure, derived as instantiations of the generic geometric structure and the generic logical structure descriptions for the class of documents considered. In figure 1 an example document is shown with in figure 2 the corresponding geometric and logical structure tree.

\subsection{Document Analysis Tools}

The construction of hyperdocuments from paper originals requires a number of modules, which are indicated in figure 3 and described here.

First, the document is scanned converting it into a digital image. We only consider binary scanning where each pixel in the digital image indicates whether it is part of the foreground or the background. Pixels identified as foreground will contain the characters, lines in graphical pictures etc, whereas the background will contain all "white space" in the document. This configuration of black and white pixels implicitly contains the document structure. The aim of document structure analysis is to extract the structure of the document from its digital image. To be precise, we aim to find the specific geometric and logical tree corresponding to the original paper document. From there, the analysis continues by analyzing the content of the text blocks using OCR, keyword search (in the text) and label search in (text and/or picture). We will first discuss how to extract the implicit ODA trees.

Various methods for document structure analysis are known in literature (e.g. [9]). Our method is a bottom-up method based on the bounding boxes of connected components in the image rather than the run-length coding of the image as used in the reference. In 


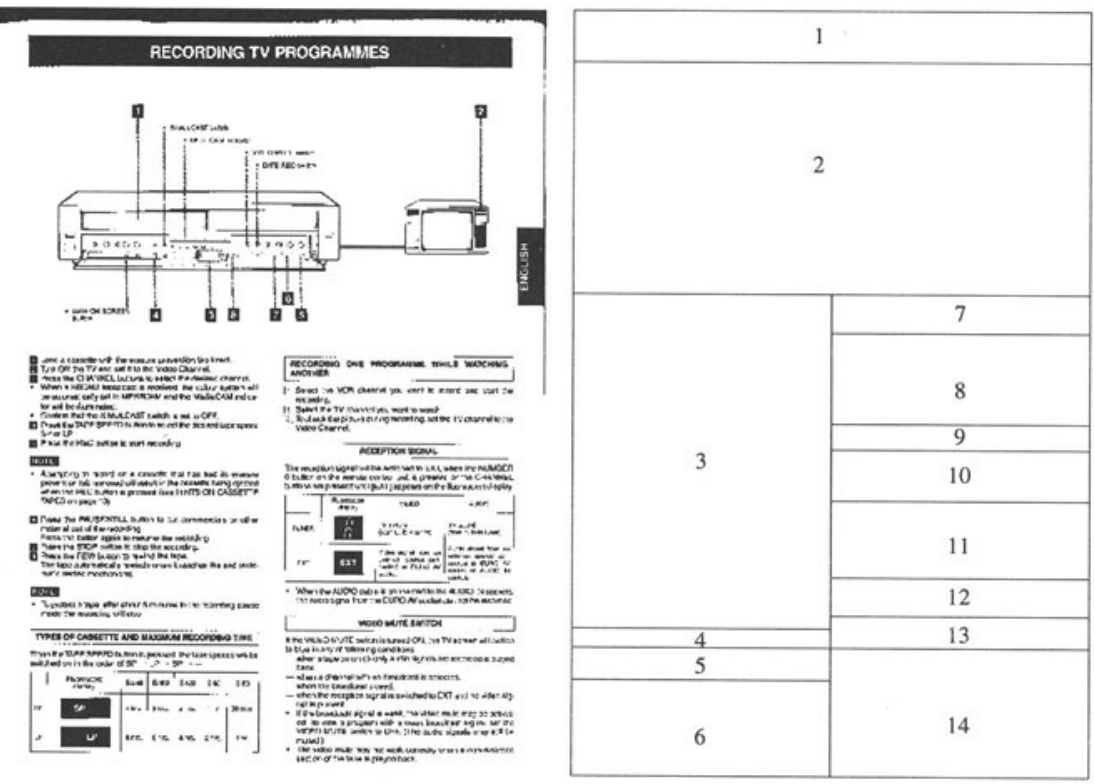

Figure 1 An example of a structured document and the geometric blocks corresponding to the coherent parts of the document.

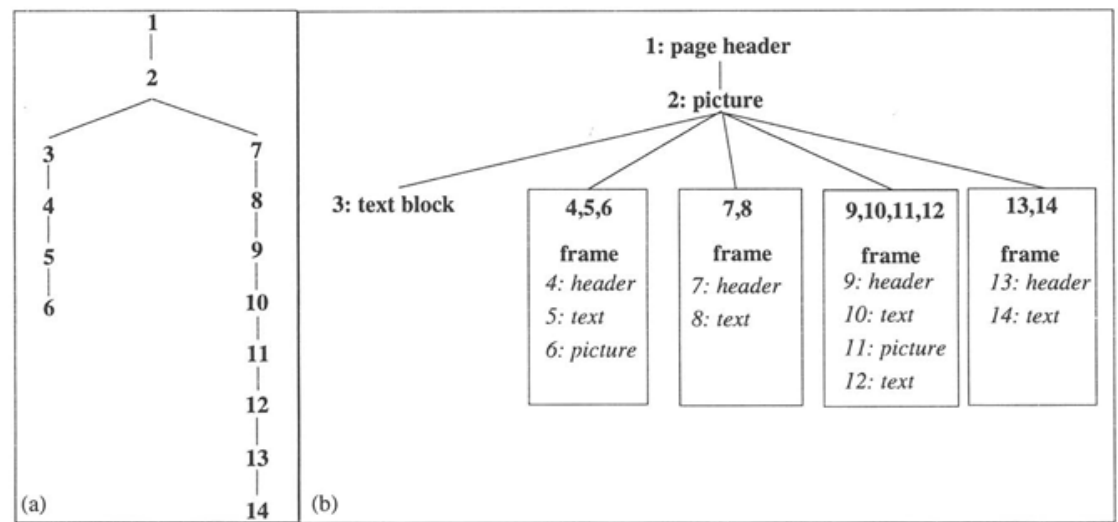

Figure 2 The specific geometric tree corresponding to the example in figure 1 (a) and its corresponding specific logical tree (b). 


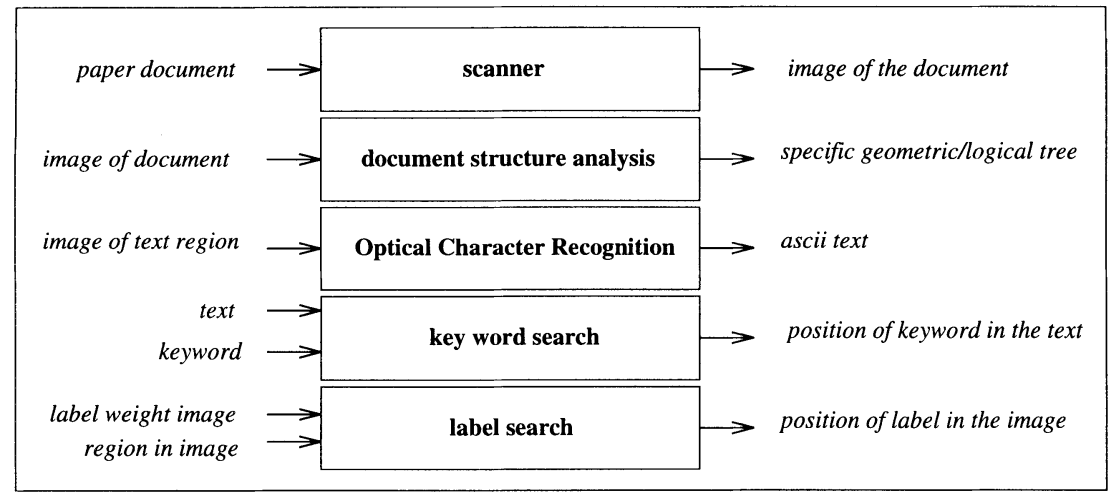

Figure 3 Overview of the different modules which are needed to perform automated hypertext construction.

the first step, objects in the image are extracted where an object is classified as being text, a picture, or a vertical/horizontal line in the image based on simple properties of the bounding box and the pixels within the box. From there, single characters and word fragments are combined into lines of text which are subsequently merged into paragraphs. These text blocks together with the picture blocks generate the specific geometric tree. As the method is very general it gives good results for a large class of documents with simple block structure. Given the specific geometric structure the logical structure can be created manually or using a number of rules for tree transformation as used in [9]. The latter method is useful for newspaper type of documents and is specifically aimed at establishing the reading order of the document. When the document structure is too complicated to handle, we use manual zoning and let the user define the trees interactively.

When documents have a complicated, but generic, structure as is the case in manuals of a specific company or patent applications in a particular domain one can use a method which exploits generic ODA structures [2]. This is a powerful top-down method in which a number of generic ODA trees in a hierarchical fashion are matched to a given document. This method is not included in the current prototype system.

Any of the above methods leads to a tree structure where some blocks are classified as picture blocks and some are classified as text blocks. The text blocks are sent to the $O C R$ Module. This module takes a digital image of a text region, recognizes the individual characters, and returns the text in ASCII form. This step also involves lexical analysis to improve the quality of character recognition on the basis of the local context.

Having retrieved the text in a document in ASCII form we can search for specific words in the text. This keyword search returns the position of the given keyword in the text.

The final module in the system deals with blocks in the image of the document classified as pictures. To be precise, given a digital image of a detail $l$ present in the picture block, we seek to find the position(s) at which this particular detail is found. This detail detection is done using the Enigma-system [3]. This is a system in which the user defines a binary template by extracting it as part of an example picture. From there, the user can edit the 
template and assign weights to different parts of the template. Positive weights indicate the importance of an object pixel to be present, whereas a negative part indicates the importance of some background pixel. Finally, the value 0 indicates a "don't care" region. A number of alternative strategies for automatic weight assignment are available where for example one can put more emphasis on pixels located on the centerline of the object (or the background) then to the contour of the template. This is to allow for some noise in the object boundaries.

Using fuzzy mathematical morphology the template is searched for in the picture and at each position a confidence value $\operatorname{conf}(l) \in[-100,100]$ is derived indicating the correspondence of the template specification and the local pixel configuration. The value $\operatorname{conf}(l)$ can be interpreted as the (weighted) percentage of points in the local configuration in the picture which are of the same value as the template (which only contains the values 0 and 1) [10]. Local positive maxima of this confidence field are the candidate positions for the detail in the picture. In practice a threshold $t$ on the confidence is applied to limit the number of candidates. For more complex picture details one can decompose the detail into several parts, locating them separately in the picture. Candidates found this way are combined to find the full detail subject to a set of strict topological relations (left-of, above etc) specified by the user.

The set of tools described above and summarized in figure 3 form the basis for the automated hypertext construction.

\section{$3 \quad$ LINKS}

In the previous section we considered the structure of the document and the contents of individual blocks. The essence of hypertext generation is creating links between these pieces of information. In order to propose hyperlinks to add to the hypertext we first consider the different types of links one encounters in a document and from there introduce methods, based on the modules described in the previous section, to find the various links.

\subsection{Types of links}

Commonly known links are $<$ text $>-<$ text $>$ links. They link words in one document to related words in another document (part). This can be the same word found in both of the documents, or the two words can be different, but then they must have a semantic relation. The latter type of links will not be considered here, but methods for detecting them can be found in [7].

In a document with pictures it is important to define $\langle$ picture $\rangle-\langle$ text $\rangle$ links, where the picture can either be a photograph or graphics. Here the link can either be to the picture itself or to its caption.

The third class of links we consider are links between parts of a picture and the text. To be more precise we define $<$ picture label $>-<$ text $>$ links where the picture label can be one of the following three types:

1. <generic text label $>$ : Label with a generic shape containing a character string.

2. <plain text label>: Isolated character string in the picture. 


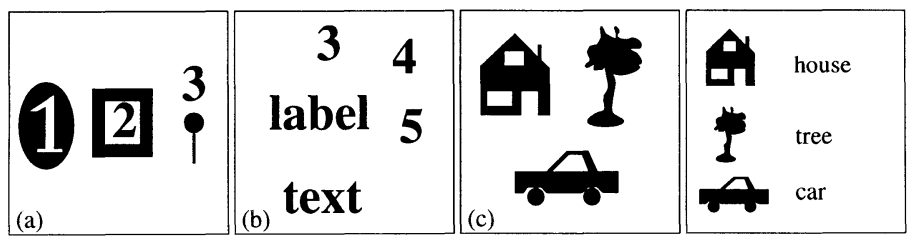

Figure 4 Examples of picture labels. (a) Generic text labels, (b) plain text labels (c) icon labels and (d) labels in a legend.

3. <icon label>: Label distinguished by its shape only.

In figure 4 some example picture labels are shown.

When considering links between a picture label and the text the above classes lead to $3 \times 3$ possible combinations. However, in general documents not all of them will occur. First, and most important, observation is that generic text and icon labels seldom occur in the text when there are no labels of corresponding type present in the picture. Furthermore, icon labels in the picture which have no label in the text are only useful if a semantic description of the label is present. This is the case only when a legend with <icon label $>-$ $<$ string $>$ pairs is present in the paper document.

\subsection{Automated link definition}

We first consider $<$ text $>$-<text $>$ links. A simple automatic link definition is on the basis of user defined keywords. In the output of the OCR-system the keywords are detected and links between keywords detected in different document parts are suggested.

Specific logical parts of the document like the index, the contents page, and footnotes provide the information to define the keyword to search for automatically, based again on the OCR-result. The specific logical class also defines the region in which the keywords are to be found. For example in the case of a footnote we have to restrict the search to the page on which it is located as very often the numbering of footnotes is reset at every page. Furthermore, when analyzing the index we already know at which page to find the keyword.

Automatic $<$ picture $>-<$ text $>$ links are found by locating the caption of the picture and then using the OCR result of the caption to find the general picture indication like figure. $n n$. This keyword, including its sequence number $n n$, is searched for in the text and a link is suggested.

The final class of links to analyze are the $<$ picture label $>-<$ text $>$ links. The methods to find those are all based on the modules as described earlier. They differ in the order of applying the modules and the region of the image of the documents to which the modules are applied.

As concerns the number of steps in automatic link definition the easiest of the links to find is the <icon label $>$-<icon label $>$ link. In this case the following steps are performed: 


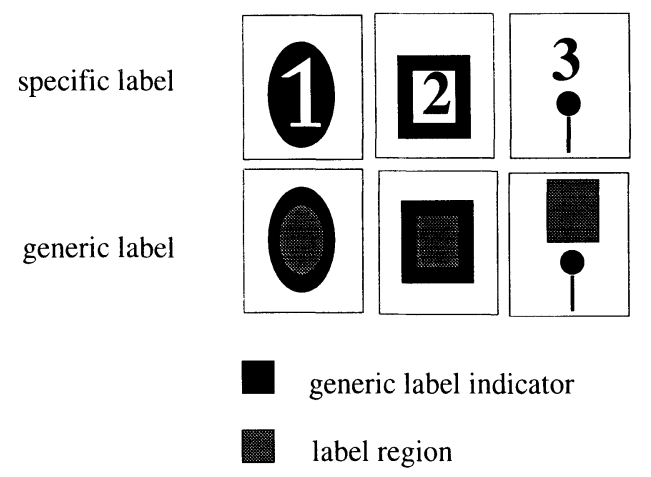

Figure 5 In the top part of the figure some specific labels are shown. In the lower part of the figure the generic part of the label is indicated as well as the part of the label which encloses the label identifier (the label region).

1. Label definition: the user interactively creates a label by using either the label in the image or the label in the image of the text.

2. Label search: the label is searched for in the corresponding text or picture.

The second case we consider is the case where a generic text label is present in both the picture and the text. For such a label we have to distinguish the generic part of the label, which is the same for all labels, and the label region in which the text is located which is different for each instantiation of the generic label. In the construction of a weight function for label detection the label region is set to "don't care" values. The difference between the generic part of a label and its label region is illustrated in figure 5 .

As sometimes the labels in the text and the picture are of different size we treat the two sets of labels separately. For both sets we go through the following steps:

1. Label definition specification of the generic part of the label and the label region.

2. Label search in text and picture

3. $O C R$ of the label region in each detected label.

4. Link definition on the basis of the output of the OCR-module.

In many documents the generic label is used in the picture only and in the corresponding text the character string contained in the label region is used. In this case a keyword search with the recognized contents of the label region is performed.

Doing a search for plain text labels in the picture is a difficult task as the character strings in the picture do not have any generic part identifying them as labels. In the case of numerals, assuming they are all defined in the same font, we define an example template for all 10 symbols. This is feasible if many numeric labels are present in the picture which is for example the case in technical drawings. In principle this could be 
done for the 26 characters as well, but often interactive location of the string will prove to be faster and more accurate. The method leads to the following steps:

\section{Label definition}

2. Label search in the picture

3. Numeral grouping of detected numerals which are close together and either oriented in a horizontal or vertical fashion.

4. $O C R$ of the label in the picture

5. Keyword search in the text

The final links to find are the ones for which there is a legend attached to the picture. In that case the following analysis method is used:

1. Legend analysis to detect the icon label, description pairs

2. Label search in the picture

3. $O C R$ of icon description to identify corresponding text string

4. Keyword search in the text

Note that in all cases where OCR is performed on a piece of text we have a validation of the label detection in the sense that if the OCR module does not recognize the text we presumably have detected a wrong label.

With the above techniques hyperlinks can be suggested. After confirmation by the user they are added to the hypertext. Proceeding in this manner a complete hypertext document is created. In the next section we consider the storage and the retrieval of such documents.

\section{AN ODA/DEXTER HYPERDOCUMENT}

We designed a simple browsing and storage system using the data structures of the recently developed Dexter Hypertext Reference Model; a standard for comparison and design of hypertext systems [4] in which we incorporated the ODA structures. In figure 6 an overview of the data structure with embedded ODA is given.

The basic items in a Dexter system are the atoms, where an atom can for example be a picture, a piece of text (or even a video) etc. These atoms can be combined into composites, which in turn might be part of another composite. The only restriction is that no composite can contain itself either directly or transitively.

Links in Dexter are between atoms and/or composites. The atoms, composites, and their relations are part of the storage layer of Dexter. This storage layer does not have any knowledge of the structure and meaning of the different components and this is one of the most important aspects of the Dexter model. The structure and meaning of the components is defined in the within-component layer. In this layer we can put the ODAspecific knowledge. The interface between the two layers is by means of anchors. Every atom or composite component can have a series of anchors defining a specific part of its contents. Each anchor has an ID which is unique within the scope of the component 


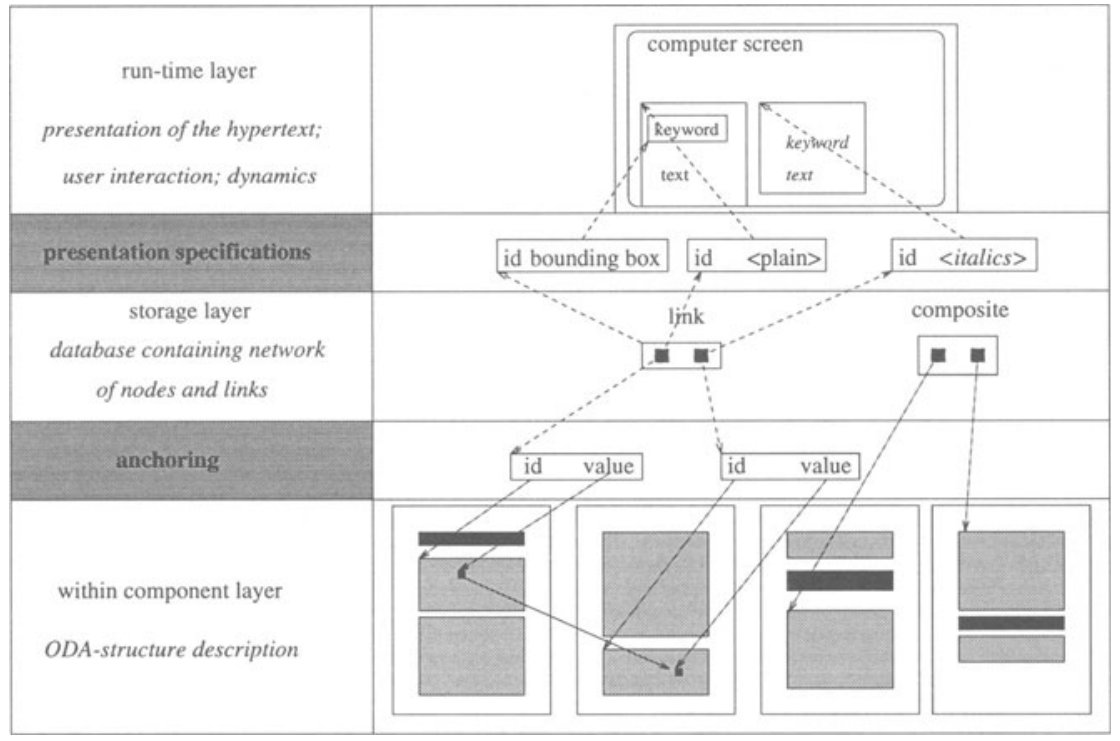

Figure 6 Incorporation of ODA into the Dexter model.

in which it is defined. It also has an associated value. This value can have an arbitrary interpretation like the $(x, y)$-position within the image of the document, or a pointer to some word in the text. This interpretation can be done by the within-component layer only. However, the storage layer can uniquely address the anchor by giving the component ID and the anchor ID. In this way the document structure analysis module can for example adjust the found position of a label, without notifying the storage layer. The latter still refers to the same anchor.

Each link between (parts of) components is described by two specifiers one for the beginning and one for the endpoint of the link. If needed one can also use multiway links by using more specifiers. Each specifier contains the following elements:

- ComponentSpec : specification of the component to link to.

- AnchorID: when the endpoint is not the component but located within its content.

- Direction: either TO, FROM, BIDIRECT, or NONE.

- PresentationSpec: what should be done if the "specifier" is activated?

Each component also has its own presentation specification telling how it should be presented to the user. The hypertext system has to make its final decision on the basis of the link used to arrive at the component and the the presentation specification of the component itself. A component can further have an arbitrary set of attribute-value pairs in which component information can be stored.

Considering the previous description it is relatively easy to define a ODA hyperdocu- 
ment within the Dexter data structures. Page-sets, pages and frames are mapped directly to composites in Dexter, whereas the basic geometric/logical objects are the atoms. Specific object information is stored by making use of attributes.

It should be noted that in general it is sufficient to store the logical tree only. For example a section which is divided over two pages should be presented to the user as one composite component.

The automatically derived linkages as discussed in the previous section are stored by making use of anchors, where the anchor-value is an index into a text if the endpoint is a word and it points to a bounding box in the image if it is a (generic) text label or an icon label.

\section{$5 \quad$ RESULTS}

In this section we show some results of the method of hyperlink acquisition for generic text labels and icon labels. The example documents are both manuals; one for a VCR and one for a television (in dutch).

The result for the VCR manual is shown in figure 7. In this example the generic part of the text label was defined interactively in both the image of the VCR as well as in the image of the corresponding text region. From there, the labels were searched for in both images. In both cases all genuine labels were found as the top candidates using the label search module. The label region of each label found was sent to the OCR-module and on the basis of this result the linkages between the picture and the corresponding text were defined. The corresponding Dexter data structure for the link indicated is shown in figure 8 .

For the second example we defined an icon label in the picture shown in figure 9 and used it to find the label in the associated text. Again the labels to be found were returned as the top candidates by the label search module.

\section{CONCLUSION}

In this paper we described a prototype system which starts of with a paper document and goes through a number of steps to generate the underlying hypertext conveyed by the document. First, the document is scanned leading to a digital binary image of the document. Then the structure of the document is analyzed. The Open Document Architecture makes a distinction between the geometric and logical structure of the document. This is precisely what is used in the document analysis step, first the geometric structure is found by looking at the way the document is presented and this result is used to generate the logical structure in which each block in the image of the document is given a meaning. Text blocks are then analyzed using OCR which in turn is the basis for finding keywords in the text. In the picture blocks one can automatically find picture details by using a sophisticated form of template matching. We have analyzed the various types of links one can encounter in general documents and for all generic links we have designed algorithms for hyperlink suggestion based on the limited set of modules described in the paper.

Hyperdocuments can have a very complicated structure. Both in their geometric and 


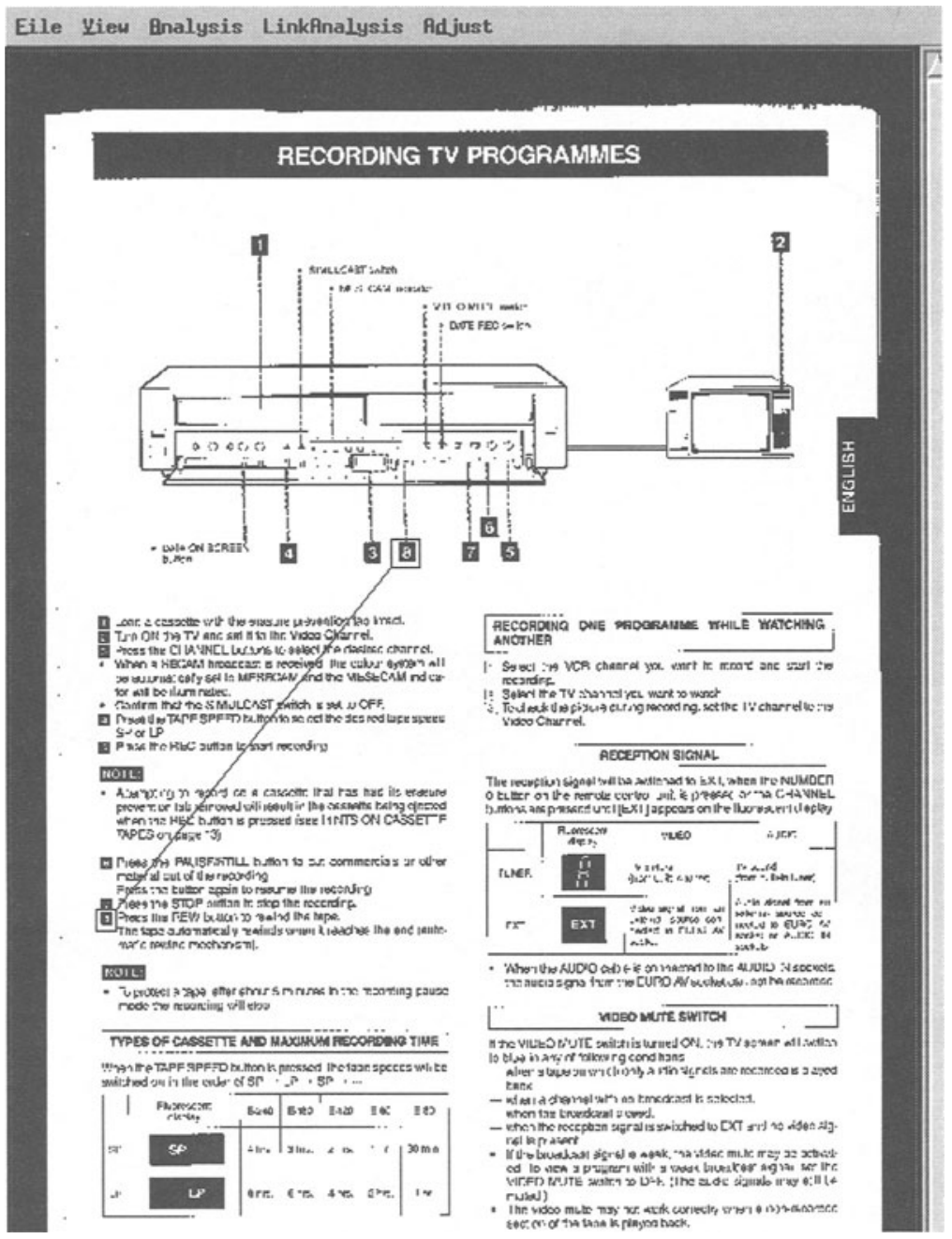

Figure 7 Example of a document containing generic text labels in both the picture and the text. The link between the instantiations corresponding to the textual label 8 is indicated. 


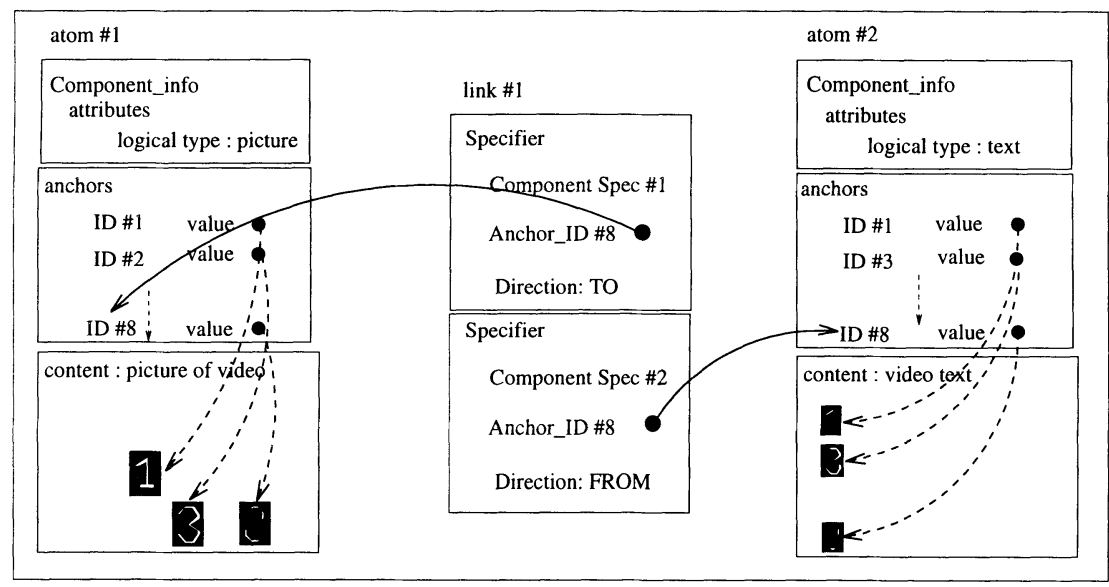

Figure 8 Example of a link in the Dexter structure for the example given in figure 7

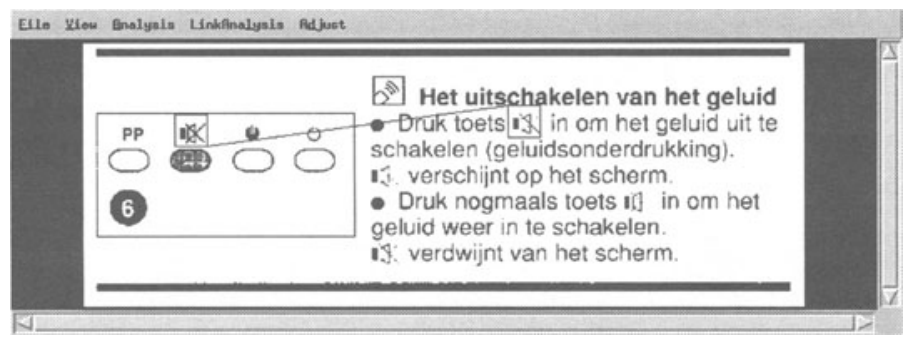

Figure 9 Example of an icon label found in the image and linked to the same icon label found on the second line of the text. This specific line of text (in Dutch) states "push button <icon>".

logical structure as well as in the structure of hyperlinks. We have therefore designed data structures combining the ODA-structure, which is the most convenient way of describing the structure of a document, with the Dexter Reference model being the appropriate model for the structure of the hyperlinks.

Although we only showed a limited set of examples of automatic hyperlinks between picture details and the text they indeed are very promising. Current research is devoted to a more elaborate evaluation of the automated linkage methods.

\section{Acknowledgment}

We thank Theo Gevers for providing us with the Enigma system. 


\section{REFERENCES}

I.R. Campbell-Grant. Introducing ODA. Computer Standards \& Interfaces, 11:149-157, 1991.

A. Dengel. From paper to office document standard representation. IEEE Computer, 25(7):63-67, 1992.

T. Gevers and A.W.M. Smeulders. Indexing of images by pictorial information. In Proceedings of Visual Database Systems II, Budapest, pages 93-100, 1991.

F. Halasz and M. Schwartz. The Dexter hypertext reference model. Communications of the ACM, 37(2):30-39, 1994.

A. Myka and U. Güntzer. Using electronic facsimiles of documents for automatic reconstruction of underlying hypertext structures. In Second International Conference on Document Analysis and Recognition, Tsukuba Science City, Japan, pages 528-532, 1993.

G. Nagy, S. Seth, and M. Viswanathan. A prototype document image analysis system for technical journals. IEEE computer, 25(7):10-22, 1992.

K. Parsaye, M. Chignell, S. Khosahfian, and H. Wong. Intelligent databases; objectoriented, deductive hypermedia technologies. Wiley, 1989.

S. Satoh, A. Takasu, and E. Katsura. A collaborative approach supporting method between document processing and hypertext construction. In Second International Conference on Document Analysis and Recognition, Tsukuba Science City, Japan, pages 533-536, 1993.

S. Tsujimoto and H. Asada. Major components of a complete text reading system. Proceedings of the IEEE, 80(7):1133-1149, 1992.

R. Boomgaard van den. Threshold logic and mathematical morphology. In Proceedings of the 5th International Conference on Image Analysis and Processing, Positano, Italy, pages 111-118, 1989.

\section{BIOGRAPHY}

Marcel Worring received his M.Sc. degree in computer science (with honors) from the Free University Amsterdam in 1988 and his Ph.D. degree from the University of Amsterdam in 1993. Currently he holds a post-doc position at the University of Amsterdam on document image analysis and model based video analysis.

Rene Buitenhuis was a Masters student at the University of Amsterdam and designed and implemented part of the described prototype system.

Arnold W.M. Smeulders is professor of Computer Science on Multi Media Information Systems. He has been in image processing since 1977 when he completed his MSc in physics from Delft University of Technology. His current interest is in image databases and intelligent interactive image analysis systems, as well as method- and system engineering aspects of image processing especially for documents and geographical information. 